\title{
MS03-P02 | THE MORPHEUS PROTEIN CRYSTALLISATION SCREENS
}

Gorrec, Fabrice (MRC Laboratory of Molecular Biology, Cambridge, GBR)

Three MORPHEUS 96-condition protein crystallisation screens have been formulated de novo to solve novel structures of proteins and macromolecular complexes. Each MORPHEUS screen is a 3D grid where 8 mixes of additives are combined with 4 cryoprotected precipitant mixes and 3 buffer systems (Fig. 1). Systematic approaches were followed to select the reagents and formulate the screens, like the integration of ligands that are found in crystal structures of the Protein Data Bank (PDB). Additives that can act as protein stabilisers, crystal cross-linkers, etc. were integrated to increase the yield of quality diffraction crystals. To formulate the latest screen (MORPHEUS III), small drug-like compounds as additives were integrated (Fig. 1). In addition, pragmatic aspects that enable the steps towards structure solution were taken into consideration. For example, cryoprotectants are integrated for straightforward flash-freezing of crystals. In order to avoid biases towards a specific subset/class of targets, the formulation prototypes were tested on a broad range of samples purified at the LMB.

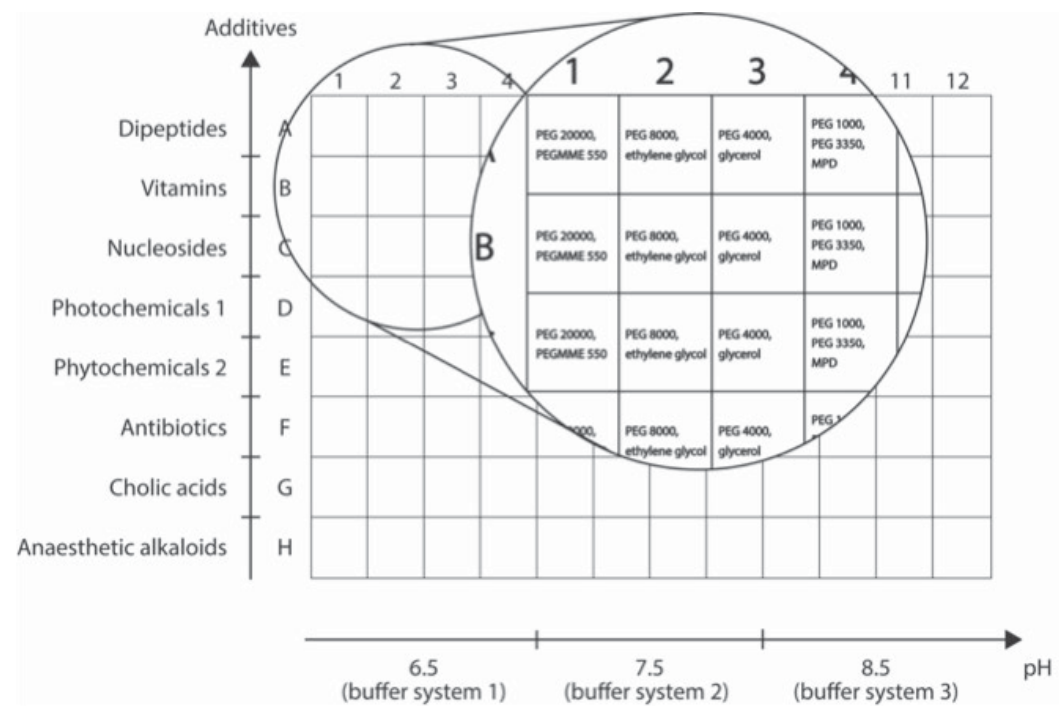

Figure 1. MORPHEUS III is a 3D grid screen composed of cryoprotecting precipitant mixes (e.g. PEG 4000 with glycerol), 3 buffer systems (pH 6.5, 7.5 and 8.5) and 8 additive mixes (Dipeptides, Vitamins, Nucleosides, etc.)

[1] Gorrec, F. (2009) The Morpheus protein crystallisation screen. J Appl Cryst 7, 1035-1042.

[2] Gorrec, F. (2015) The Morpheus II protein crystallisation screen. Acta Cryst F71, 831-837.

[3] Gorrec, F. and Zinzalla, G. (2018) The MORPHEUS III protein crystallization screen: at the frontier of drug discovery. IUCr Newsletter 26. 